\title{
ON THE DISCRIMINANT OF ARBITRARY ALGEBRAIC NUMBER FIELDS ${ }^{1}$
}

\section{J. M. CALloWAY}

One of the important applications of Minkowski's Lemma on linear forms is the proof of the fact that the discriminant $d$ of every algebraic number field of degree $n \geqq 2$ is divisible by at least one prime number.

A beautiful identity of C. L. Siegel gave this result immediately for totally real fields. ${ }^{2}$ In Siegel's paper he notes that analogous developments are valid for the general case. In this paper we shall obtain a generalization of Siegel's identity which yields the

THEOREM. The absolute value of the discriminant $d$ of an arbitrary algebraic number field $K$ of degree $n \geqq 2$ is always greater than $(\pi / 3)^{2 r_{2}}$ $\left(n=r_{1}+2 r_{2}\right)$.

Let $K$ be an arbitrary algebraic number field of degree $n \geqq 1$. Its discriminant, $d$, is a rational integer, $|d| \geqq 1$. If $n=r_{1}+2 r_{2}$, we denote the $n$ conjugates of any number $\mu \in K$ by $\mu^{(1)}, \mu^{(2)}, \cdots, \mu^{(n)}$ where $\mu^{(1)}, \mu^{(2)}, \cdots, \mu^{\left(r_{1}\right)}$ are always real and $\mu^{\left(r_{1}+\nu\right)}$ is the complex conjugate of $\mu^{\left(r_{1}+r_{2}+\nu\right)}, \nu=1,2, \cdots, r_{2}$. If $\omega_{1}, \omega_{2}, \cdots, \omega_{n}$ form a basis for $K$, then the reciprocal matrix of $\left(\omega_{l}^{(\mathbf{k})}\right)(k=1,2, \cdots, n$; $l=1,2, \cdots, n)$, we shall designate by $\left(\Omega_{k}^{(l)}\right)$. It is known that $\Omega_{1}, \Omega_{2}, \cdots, \Omega_{n}$ is then a basis for the fractional ideal $1 / \mathfrak{b} \subset K$. The integral ideal $b$ is called the different or Fundamental Ideal and has norm equal to the absolute value of the discriminant $d$ of $K$.

In all that follows, whenever we encounter a product $\Pi_{l}$, or a sum $\sum_{l}$, we understand the quantities $\lambda, \mu, \xi, \eta, \theta, x, y, z$ to have superscripts $\lambda^{(l)}, \mu^{(l)}, \xi^{(l)}, \eta^{(l)}, \theta^{(l)}, x^{(l)}, y^{(l)}, z^{(l)}$.

Our theorem about the discriminant, $d$, now follows from the identity

(1) $\left|d^{1 / 2}\right|=\left(\frac{\pi}{3}\right)^{r_{2}}+(2 \pi)^{r_{2}} \sum_{(1 / b) \mid \lambda}^{\prime} \prod_{l=1}^{r_{1}}\left(\frac{\sin \pi \lambda}{\pi \lambda}\right)^{2} \cdot \prod_{l=r_{1}+1}^{r_{1}+r_{2}} G(2 \pi|\lambda|)$,

where $\lambda$ runs through all the elements of the ideal $1 / \delta$ except 0 , and

Presented to the Society, December 29, 1952; received by the editors August 9, 1954.

1 Presented to the Graduate School of the University of Pennsylvania in partial fulfillment of the requirements for the degree of Doctor of Philosophy.

${ }^{2}$ Göttingen Nachrichtin (1922) pp. 17-24. 


$$
G(x)=\sum_{m=0}^{\infty} \frac{(-1)^{m} x^{2 m}}{(2 m+3) m !(m+2) !} .
$$

It will be shown that $G(2 \pi|\lambda|)>0$ for all $\lambda$.

When $n=r_{1}+2 r_{2}, r_{2} \neq 0, d$ is obviously greater than 1 . If $r_{2}=0$, then (1) becomes Siegel's formula

$$
\left|d^{1 / 2}\right|=1+\sum_{(1 / b) \mid \lambda}^{\prime} \prod_{l=1}^{n}\left(\frac{\sin \pi \lambda}{\pi \lambda}\right)^{2} .
$$

If $n=1$, this merely says that $d=1$, but if $n>1$, then there exists $\lambda \in 1 / b$ not a rational integer and the sum in (1a) is not equal to 0 . The result follows.

It may be remarked that the fact that the right-hand side of (1a) converges is rather remarkable. In most fields $\sum_{(1 / b) \mid \lambda}^{\prime} \prod_{l=1}^{n}(1 / \pi \lambda)^{2}$ diverges, since there are in general infinitely many units in an algebraic number field. The convergence of this sum and the others appearing in (1) and (2) will be shown in the course of the paper.

(1) is a specialization of the identity

$$
\begin{aligned}
& \sum_{a|\mu,| \mu \mid \prec x} \prod_{l=1}^{n} e^{2 \pi i \mu \theta}(x-|\mu|)^{k} \\
(2)= & \frac{2^{n} \pi^{r 2}}{N a\left|d^{1 / 2}\right|} \sum_{(1 / a b) \mid \lambda} \prod_{l=1}^{r_{1}} k !\left(\sum_{\nu=1}^{[(k+1) / 2]} \frac{(-1)^{r-1} x^{k-2 \nu+1}}{(k-2 \nu+1) !\{2 \pi(\theta-\lambda)\}^{2 v}}\right. \\
& \left.+\frac{(-1)^{[(k+1) / 2]}}{\{2 \pi(\theta-\lambda)\}^{k+1}} \sin \left\{2 \pi(\theta-\lambda) x+\delta \frac{\pi}{2}\right\}\right) \\
& \prod_{l=r_{1}+1}^{r_{1}+r_{2}} \int_{0}^{x}(x-r)^{2 k_{r}} J_{0}(4 \pi|\theta-\lambda| r) d r,
\end{aligned}
$$

where the ideal $a \subset K ; x^{(l)}, l=1,2, \cdots, n$, are positive real numbers with $x^{r_{1}+\nu}=x^{r_{1}+r_{2}+\nu}, \nu=1,2, \cdots, r_{2} ; \theta^{(l)}, l=1,2, \cdots, n$, are $n$ numbers such that $\theta^{(1)}, \theta^{(2)}, \cdots, \theta^{\left(r_{1}\right)}$ are real and $\theta^{\left(r_{1}+\nu\right)}=\bar{\theta}^{\left(r_{1}+r_{2}+\nu\right)}$, $\nu=1,2, \cdots, r_{2}$; and $\theta^{(l)}$ have been so chosen that $\theta^{(l)}-\lambda^{(l)} \neq 0$ for $\lambda \in 1 / \mathfrak{a} \delta ; k$ is a positive integer; $|\mu| \prec x$ means that we take only those $\mu \in \mathfrak{a}$ for which the $n$ differences $x^{(l)}-\left|\mu^{(l)}\right|>0, l=1,2, \cdots, n$; $\delta=0$ for $k$ even and $\delta=1$ for $k$ odd.

The case when $\theta^{(l)}-\lambda^{(l)}=0$ is considered separately below and in this case a different expression must be taken for the $C_{\lambda}$ in (7).

An interesting result is then obtained by setting $K=R(1) ; n=1$; $a=(1) ; \theta^{(1)}=0$; and restricting $x$ so that $0<x \leqq N a=1$. (This result follows easily from (9) below.) 


$$
\begin{aligned}
x^{k}=\frac{2 x^{k+1}}{k+1} & +4 \sum_{l=1}^{\infty}\left(\sum_{\nu=1}^{[(k+1) / 2]} \frac{(-1)^{\nu-1} k ! x^{k-2 \nu+1}}{(k-2 \nu+1) !(2 \pi l)^{2 \nu}}\right. \\
+ & \left.\frac{(-1)^{[(k+1) / 2]} k !}{(2 \pi l)^{k+1}} \sin \left(2 \pi l x+\delta \frac{\pi}{2}\right)\right), \quad 0<x \leqq 1 .
\end{aligned}
$$

The equation ( 3 ) is equivalent to the well known Fourier expansion for the $(k+1)$ st Bernoulli Polynomial

$$
\frac{1}{(k+1) !}(x+B)^{k+1}=\left\{\begin{array}{lr}
(-1)^{m-1} \sum_{l=1}^{\infty} \frac{2 \sin 2 \pi l x}{(2 \pi l)^{2 m+1}}=P_{2 m+1}(x), & k=2 m, \\
(-1)^{m-1} \sum_{l=1}^{\infty} \frac{2 \cos 2 \pi l x}{(2 \pi l)^{2 m+2}}=P_{2 m+2}(x), & k=2 m+1,
\end{array}\right.
$$

where $(x+B)^{k+1}$ is interpreted symbolically.

The identity (1) may be obtained in either of two ways. One is a direct analogue of C. L. Siegel's method for totally real fields. This does not give the more general identity (2).but upon specialization does yield (1), and consequently, the result about the discriminant. The other method which follows yields (2) directly.

We derive (2) by expanding a certain function $f(t)=f\left(t_{1}, t_{2}, \cdots, t_{n}\right)$ in an $n$-fold Fourier series.

Let $\left(\alpha_{1}, \alpha_{2}, \cdots, \alpha_{n}\right)$ be a basis for the ideal $\mathfrak{a} \subset K$.

Let $t_{1}, t_{2}, \cdots, t_{n}$ be $n$ real variables.

Let $x^{(1)}, x^{(2)}, \cdots, x^{(n)}$ be $n$ fixed positive real numbers with $x^{\left(r_{1}+\nu\right)}=x^{\left(r_{1}+r_{2}+\nu\right)}, \nu=1,2, \cdots, r_{2}$.

Let $\theta^{(1)}, \theta^{(2)}, \cdots, \theta^{(n)}$ be $n$ numbers such that $\theta^{(1)}, \theta^{(2)}, \cdots, \theta^{\left(r_{1}\right)}$ are real and $\theta^{\left(r_{1}+\nu\right)}=\bar{\theta}^{\left(r_{1}+r_{2}+\nu\right)}, \nu=1,2, \cdots, r_{2}$.

We define $\xi^{(l)}=t_{1} \alpha_{1}^{(l)}+t_{2} \alpha_{2}^{(l)}+\cdots+t_{n} \alpha_{n}^{(l)} ; l=1,2, \cdots, n$.

For every $\mu \in \mathfrak{a}$, we set $\mu^{(l)}=m_{1} \alpha_{1}^{(l)}+m_{2} \alpha_{2}^{(l)}+\cdots+m_{n} \alpha_{n}^{(l)}$; $l=1,2, \cdots, n$.

The function $f(t)=f\left(t_{1}, t_{2}, \cdots, t_{n}\right)$ is then defined as the finite sum

$$
f(t)=\sum_{a|\mu,| \mu+\xi \mid} \prod_{l=1}^{n}(x-|\mu+\xi|)^{k} \exp (2 \pi i(\mu+\xi) \theta)
$$

where $|\mu+\xi|<x$ means that the sum extends over only those elements for which $x^{(l)}-\left|\mu^{(l)}+\xi^{(l)}\right|>0, l=1,2, \cdots, n ; k$ being a positive integer. Hereafter all summations over $\mu$ will be understood to be subject to this restriction.

The function $f\left(t_{1}, t_{2}, \cdots, t_{n}\right)$ is obviously periodic in $t_{1}, t_{2}, \cdots, t_{n}$ with period 1. Further $f(t)$ has derivatives of every order at all points of $t_{1} \cdots t_{n}$ space except possibly at the lattice points and points 
where $\left|\mu^{(l)}+\xi^{(l)}\right|=0$ for some $l$, and even at these points as a function of each separate variable, left- and right-hand derivatives exist and are bounded. Consequently $i_{i}$ the fundamental hypercube $\left(-1 / 2 \leqq t_{1} \leqq 1 / 2, \cdots,-1 / 2 \leqq t_{n} \leqq 1 / 2\right), f(t)$ is of bounded variation and has bounded partial derivatives of the first order. By a theorem of Tonelli, ${ }^{3}$ the $n$-fold Fourier series associated with $f(t)$ converges to the function in the sense of Stolz and Pringsheim. ${ }^{4}$ That is, if we define the partial sum $s_{a_{1} \ldots a_{n}}\left(t_{1}, \cdots, t_{n}\right)$ to be

$$
\sum_{q_{1}=-a_{1}}^{a_{1}} \cdots \sum_{q_{n}=-a_{n}}^{a_{n}} C_{q_{1} \cdots q_{n}} \exp \left(2 \pi i\left(q_{1} t_{1}+\cdots+q_{n} t_{n}\right)\right),
$$

where the $C_{q_{1}} \cdots_{q_{n}}$ are coefficients of the $n$-fold Fourier series, we say that the series converges to the sum $S\left(t_{1}, \cdots, t_{n}\right)$ if given $\epsilon>0$, it is always possible to determine $N>0$, such that for any choice of $a_{1}, \cdots, a_{n}$ such that each $a_{i}>N, \mid S\left(t_{1}, \cdots, t_{n}\right)-s_{a_{1} \cdots a_{n}}\left(t_{1}, \cdots\right.$, $\left.t_{n}\right) \mid<\epsilon$. From now on we always understand that the summation is to be made in this way. With this understanding, we write

$$
f(t)=\sum_{q_{1}} \cdots \sum_{q_{n}} C_{q_{1}} \cdots q_{n} \exp \left(2 \pi i\left(q_{1} t_{1}+\cdots+q_{n} t_{n}\right)\right) .
$$

Since $\left(\alpha_{1}, \alpha_{2}, \cdots, \alpha_{n}\right)$ is a basis for the ideal $a \subset K$, then the reciprocal matrix

$$
\left(A_{k}^{(l)}\right)\left(\begin{array}{l}
l=1,2, \cdots, n \\
k=1,2, \cdots, n
\end{array}\right)
$$

of the matrix $\left(\alpha_{l}^{(k)}\right)$ gives a basis $A_{1}, \cdots, A_{n}$ for the ideal $1 /$ ad .

$\lambda=q_{1} A_{1}+\cdots+q_{n} A_{n}$ runs through all the elements of $1 / \mathfrak{a d}$ if $q_{1}, \cdots, q_{n}$ run through all rational integers. Moreover

$$
\begin{aligned}
q_{1} t_{1}+\cdots+q_{n} t_{n} & =\sum_{k=1}^{n} q_{k} \sum_{l=1}^{n} \xi^{(l)} A_{k}^{(l)}=\sum_{l=1}^{n} \xi^{(l)} \sum_{k=1}^{n} q_{k} A_{k}^{(l)} \\
& =\sum_{l=1}^{n} \xi^{(l)} \lambda^{(l)} .
\end{aligned}
$$

If we now write $C_{\lambda}$ for $C_{q_{1}} \ldots q_{n}$, (4) becomes

$$
f(t)=\sum_{(1 / a b) \mid \lambda} C_{\lambda} \exp \left(2 \pi i \sum_{l=1}^{n} \xi \lambda\right)
$$

where

${ }^{3}$ L. Tonelli, Serie trigonometriche, Bologna, 1928, p. 468.

4 A. Pringsheim, Sitz. d. Bayer. Akad. d. Wiss., Math.-phys. Kl. vol. 27 (1897) pp. 101-152. 


$$
\begin{gathered}
C_{\lambda}=\int_{-1 / 2}^{1 / 2} \int_{-1 / 2}^{1 / 2} \cdots \int_{-1 / 2}^{1 / 2} f(t) \exp \left(-2 \pi i \sum_{l=1}^{n} \xi \lambda\right) d t_{1} d t_{2} \cdots d t_{n} \\
=\int_{-1 / 2}^{1 / 2} \int_{-1 / 2}^{1 / 2} \cdots \int_{-1 / 2}^{1 / 2} \sum_{a \mid \mu} \prod_{l=1}^{n}(x-|\mu+\xi|)^{k} \\
\cdot \exp (2 \pi i(\mu+\xi) \theta-2 \pi i \lambda \xi) d t_{1} d t_{2} \cdots d t_{n} .
\end{gathered}
$$

Although we use the notation $C_{\lambda}$ and sum over all the elements of the ideal $1 / \mathfrak{a} d$, the sum is always meant to be taken with respect to $q_{1}, \cdots, q_{n}$ in the manner previously indicated. We shall be able to remove this restriction on the method of summation at the end of the argument.

Since $(1 / \delta) \mid \mu \lambda, \sum_{l=1}^{n} \mu \lambda$ is a rational integer. Therefore if we replace $\exp \left(2 \pi i \sum_{l=1}^{n} \lambda \xi\right)$ by $\exp \left(2 \pi i \sum_{l=1}^{n} \lambda(\mu+\xi)\right)$ above, the value of the expression is unchanged.

$$
\begin{gathered}
C_{\lambda}=\sum_{a \mid \mu} \int_{-1 / 2}^{1 / 2} \int_{-1 / 2}^{1 / 2} \cdots \int_{-1 / 2}^{1 / 2} \prod_{l=1}^{n}(x-|\mu+\xi|)^{k} \\
\cdot \exp (2 \pi i(\mu+\xi)(\theta-\lambda)) d t_{1} d t_{2} \cdots d t_{n} .
\end{gathered}
$$

Let $\eta^{(l)}=\mu^{(l)}+\xi^{(l)}=\left(m_{1}+t_{1}\right) \alpha_{1}^{(l)}+\cdots+\left(m_{n}+t_{n}\right) \alpha_{n}^{(l)}=u_{1} \alpha_{1}^{(l)}+\cdots$ $+u_{n} \alpha_{n}^{(l)}$.

$$
\begin{aligned}
C_{\lambda} & =\sum_{m_{1}} \ldots \sum_{\substack{|\eta| \\
m_{n}}} \int_{m_{i}-1 / 2}^{m_{i}+1 / 2} \cdots \\
& \int_{m_{i}-1 / 2}^{m_{i}+1 / 2} \prod_{l=1}^{n}(x-|\eta|)^{k} \exp (2 \pi i \eta(\theta-\lambda)) d u_{1} \cdots d u_{n} \\
& =\int \underset{|\eta| \prec x}{\ldots} \int \prod_{l=1}^{n}(x-|\eta|)^{k} \exp (2 \pi i \eta(\theta-\lambda)) d u_{1} \cdots d u_{n} .
\end{aligned}
$$

We now make a change of variables, remembering that because of the numbering of the conjugates of elements of $K$ we may set

$$
\begin{array}{cr}
\eta^{(l)}=y^{(l)}, & l=1,2, \cdots, r_{1} ; \\
\eta^{(l)}=y^{(l)}+i z^{(l)}, & l=r_{1}+1, \cdots, r_{1}+r_{2} ; \\
\eta^{\left(l+r_{2}\right)}=y^{(l)}-i z^{(l)} & l=r_{1}+1, \cdots, r_{1}+r_{2} . \\
\left|\frac{\partial\left(u_{1}, u_{2}, \cdots, u_{n}\right)}{\partial\left(y^{(1)}, y^{(2)}, \cdots, y^{\left(r_{1}+r_{2}\right)}, z^{\left(r_{1}+1\right)}, \cdots, z^{\left(r_{1}+r_{2}\right)}\right)}\right|=\frac{2^{r_{2}}}{N \mathfrak{a}\left|d^{1 / 2}\right|} .
\end{array}
$$




$$
\begin{aligned}
C_{\lambda}= & \frac{2^{r_{2}}}{N a\left|d^{1 / 2}\right|} \prod_{l=1}^{r_{1}} \int_{-x}^{x}(x-|y|)^{k} \exp (2 \pi i y(\theta-\lambda)) d y \\
& \cdot \prod_{l=r_{1}+1}^{r_{1}+r_{2}} \iint_{\left(y^{2}+z^{2}\right)^{1 / 2}<x}\left(x-\left(y^{2}+z^{2}\right)^{1 / 2}\right)^{2 k} \exp (4 \pi i(u y-v z)) d y d z
\end{aligned}
$$

where $\theta-\lambda=u+i v$.

$$
C_{\lambda}=\frac{2^{r z}}{N a\left|d^{1 / 2}\right|} \prod_{l=1}^{r_{1}} 2 \int_{0}^{x}(x-y)^{k} \cos 2 \pi y(\theta-\lambda) d y
$$

$$
\cdot \prod_{l=r_{1}+1}^{r_{1}+r_{2}} \int_{0}^{x} \int_{0}^{2 \pi}(x-r)^{2 k} \exp (4 \pi i(u \cos \theta-v \sin \theta) r) r d r d \theta
$$

If $\theta-\lambda \neq 0$, then

$$
\begin{aligned}
C_{\lambda}= & \frac{2^{n} \pi^{r_{2}}}{N \mathfrak{a}\left|d^{1 / 2}\right|} \prod_{l=1}^{r_{1}}\left(\sum_{\nu=1}^{[(k+1) / 2]} \frac{(-1)^{\nu-1} k ! x^{k-2 \nu+1}}{(k-2 \nu+1) !\{2 \pi(\theta-\lambda)\}^{2 \nu}}\right. \\
& \left.+\frac{(-1)^{[(k+1) / 2]} k !}{\{2 \pi(\theta-\lambda)\}^{k+1}} \sin \left\{2 \pi(\theta-\lambda) x+\delta \frac{\pi}{2}\right\}\right) \\
& \cdot \prod_{l=r_{1}+1}^{r_{1}+r_{2}} \int_{0}^{x} r(x-r)^{2 k} J_{0}(4 \pi|\theta-\lambda| r) d r,
\end{aligned}
$$

where, as before, $\delta=0$ for $k$ even and $\delta=1$ for $k$ odd. $J_{0}$ is the Bessel function of order zero.

If we now substitute (7) in (5) we have the identity

$$
f(t)=\frac{2^{n} \pi^{r 2}}{N \mathfrak{a}\left|d^{1 / 2}\right|} \sum_{(1 / \mathrm{ab}) \mid \lambda} \exp \left(2 \pi i \sum_{l=1}^{n} \xi \lambda\right)
$$

$$
\begin{array}{ll}
\cdot \prod_{l=1}^{r}\left(\sum_{\nu=1}^{[(k+1) / 2]} \frac{(-1)^{\nu-1} k ! x^{k-2 \nu+1}}{(k-2 \nu+1) !\{2 \pi(\theta-\lambda)\}^{2 \nu}}\right. \\
\left.+\frac{(-1)^{[(k+1) / 2]} k !}{\{2 \pi(\theta-\lambda)\}^{k+1}} \sin \left\{2 \pi(\theta-\lambda) x+\delta \frac{\pi}{2}\right\}\right) \\
\cdot \prod_{l=r_{1}+1}^{r_{1}+r_{2}} \int_{0}^{x} r(x-r)^{2 k} J_{0}(4 \pi|\theta-\lambda| r) d r, & \theta-\lambda \neq 0 .
\end{array}
$$

If $\theta-\lambda=0$ in (6), then

$$
C_{\lambda}=\frac{2^{n} \pi^{r_{2}}}{N \mathfrak{a}\left|d^{1 / 2}\right|} \prod_{l=1}^{r_{1}} \frac{x^{k+1}}{k+1} \prod_{l=r_{1}+1}^{r_{1}+r_{2}} \frac{x^{2 k+2}}{(2 k+1)(2 k+2)} .
$$

Therefore if we understand the above value for $C_{\lambda}$ when $\theta-\lambda=0$, (8) 
will be good for any $\theta^{(l)}, l=1,2, \cdots, n$.

With this understanding, take $t_{1}=t_{2}=\cdots=t_{n}=0$. Then $\xi^{(l)}=0$, $l=1,2, \cdots, n$, and (8) becomes (2).

If in addition we now let $\theta^{(l)}=0, l=1,2, \cdots, n$, we have

$$
\begin{aligned}
& \sum_{\mathfrak{a} \mid \mu} \prod_{l=1}^{n}(x-|\mu|)^{k} \\
&=\frac{2^{n} \pi^{r_{2}}}{N \mathfrak{a}\left|d^{1 / 2}\right|}\left\{\frac{1}{(k+1)^{r_{1}}(2 k+1)^{r_{2}}(2 k+2)^{r_{2}}} \prod_{l=1}^{n} x^{k+1}\right. \\
&+\sum_{(1 / \mathfrak{a}) \mid \lambda}^{\prime} \prod_{l=1}^{r_{1}}\left(\sum_{\nu=1}^{[(k+1) / 2]} \frac{(-1)^{r-1} k ! x^{k-2 \nu+1}}{(k-2 \nu+1) !(2 \pi \lambda)^{2 \nu}}\right. \\
&\left.+\frac{(-1)^{[(k+1) / 2]} k !}{(2 \pi \lambda)^{k+1}} \sin \left(2 \pi \lambda x+\delta \frac{\pi}{2}\right)\right) \\
&\left.\cdot \prod_{l=r_{1}+1}^{r_{1}+r_{2}} \int_{0}^{x} r(x-r)^{2 k} J_{0}(4 \pi|\lambda| r) d r\right\}
\end{aligned}
$$

where $\sum_{\lambda}^{\prime}$ means $\lambda$ runs through all the elements of the ideal $1 /$ ad except 0 . For $k=1$, (9) becomes

$$
\begin{aligned}
\sum_{\mathfrak{a} \mid \mu} \prod_{l=1}^{n}(x-|\mu|)= & \frac{1}{N \mathfrak{a}\left|d^{1 / 2}\right|}\left\{\left(\frac{\pi}{3}\right)^{r_{2}} \prod_{l=1}^{n} x^{2}\right. \\
& +\sum_{(1 / \mathrm{a} \mathrm{b}) \mid \lambda} \prod_{l=1}^{r_{1}}\left(\frac{\sin \pi \lambda x}{\pi \lambda}\right)^{2} \\
& \left.\cdot \prod_{l=r_{1}+1}^{r_{1}+r_{2}} 4 \pi \int_{0}^{x} r(x-r)^{2} J_{0}(4 \pi|\lambda| r) d r\right\} .
\end{aligned}
$$

If we take $\mathfrak{a}=(1)$ and $x^{(l)}=1, l=1,2, \cdots, n$, in (10) we obtain (1). (1) may also be written

$$
\begin{aligned}
\left|d^{1 / 2}\right|= & \left(\frac{\pi}{3}\right)^{r_{2}}+\sum_{(1 / b) \mid \lambda}^{\prime} \prod_{l=1}^{r_{1}}\left(\frac{\sin \pi \lambda}{\pi \lambda}\right)^{2} \\
& \cdot \prod_{l=r_{1}+1}^{r_{1}+r_{2}} \frac{\pi}{(2 \pi|\lambda|)^{3}} \int_{0}^{4 \pi|\lambda|} J_{2}(z) d z .
\end{aligned}
$$

Now if it can be shown that $\int_{0}^{x} J_{2}(z) d z>0$ for all real positive $x$, our theorem on the discriminant of an arbitrary algebraic number field will be proved. When this has been shown, since all the terms in $\sum_{\lambda}^{\prime}$ will be positive, we shall then be able to remove the restriction on the method of summation over the elements of the ideal $1 / \delta$.

It follows from the recurrence formula for the Bessel functions that 


$$
\int_{0}^{x} J_{2}(z) d z=\int_{0}^{x} J_{0}(z) d z-2 J_{1}(x) .
$$

The minima for this function of $x$ will occur at every second zero of $J_{2}(z)$. We designate these by $j_{2,2 m}$ or simply $j$.

$$
\int_{0}^{x} J_{0}(z) d z=\frac{\pi x}{2}\left\{J_{0}(x) H_{0}^{\prime}(x)+J_{1}(x) H_{0}(x)\right\},
$$

where $H_{\nu}(x)$ is the Struve function of order $\nu$.

Now because of the interlacing of the zeroes of $J_{\nu}$ and $J_{\nu+1}$, the zeroes $j_{2,2 m}$ are contained between $j_{1,2 m}$ and $j_{1,2 m+1}$. However in these intervals $J_{1}(x)$ is positive.

Also since $J_{0}(z)+J_{2}(z)=(2 / z) J_{1}(z)$, at a zero of $J_{2}, J_{0}$ and $J_{1}$ have the same sign. Consequently at $j, J_{0}(j)=(2 / j) J_{1}(j)>0$. Then

$$
\text { Minimum } \begin{aligned}
\int_{0}^{x} J_{2}(z) d z & =\int_{0}^{j} J_{2}(z) d z \\
& =\frac{\pi j}{2}\left\{J_{0}(j) H_{0}^{\prime}(j)+\frac{j}{2} J_{0}(j) H_{0}(j)\right\}-j J_{0}(j) \\
& =\frac{\pi j}{2} J_{0}(j)\left\{H_{0}^{\prime}(j)+\frac{j}{2} H_{0}(j)-\frac{2}{\pi}\right\} .
\end{aligned}
$$

Using well known properties of the Struve functions, ${ }^{5}$ this can be written

$$
\operatorname{Minimum} \int_{0}^{x} J_{2}(z) d z=\frac{\pi j^{2}}{2} J_{0}(j)\left\{\frac{j}{3 \pi}-\frac{\boldsymbol{H}_{2}(j)}{2}\right\} .
$$

Since $J_{0}(j)>0, \int_{0}^{j} J_{2}(z) d z>0$ if we can show that $(j / 3 \pi)-\left(H_{2}(j) / 2\right)>0$. By using the asymptotic formulas for $\boldsymbol{H}_{2}(z),{ }^{5}$ it can be shown that $(j / 3 \pi)-\left(H_{2}(j) / 2\right)>0, j>6$. Therefore

$$
\text { Minimum } \int_{0}^{x} J_{2}(z) d z=\frac{\pi j^{2}}{2} J_{0}(j)\left\{\frac{j}{3 \pi}-\frac{H_{2}(j)}{2}\right\}>0 \quad \text { if } j>6 .
$$

But $j_{2,2}$ is approximately 8.4172441 .

Thus we have shown that the absolute value of the discriminant of an arbitrary algebraic number field of degree $n>1$ is greater than $(\pi / 3)^{2+2}$.

Acknowledgment. The author would like to express his appreciation to Professor H. A. Rademacher for suggesting the subject of this paper and for his many helpful criticisms.

\section{University of Pennsylvania}

${ }^{5}$ G. N. Watson, Theory of Bessel functions, Cambridge, 1945, pp. 328-338. 